\title{
KERATODERMA BLENORRHAGICUM IN A PATIENT WITH REITER SYNDROME
}

\author{
Valentina Dimitrova ${ }^{1}$, Valentin Valtchev ${ }^{1}$, Ivelina Yordanova ${ }^{1}$, Hristina Haidudova ${ }^{1}$, \\ Dimitar Gospodinov ${ }^{1}$, Snejana Tisheva ${ }^{2}$ \\ Department of Dermatology and Venereology, \\ ${ }^{2}$ Department of Rheumatology and Cardiology, \\ Medical University - Pleven
}

\begin{abstract}
Reiter syndrome is a systemic disorder, originally defined as a triad of arthritis, urethritis and conjunctivitis. This symptoms complex usually follows an episode of either urethritis or dysentery. Skin and mucosal involvement is observed in about $10 \%$ of the cases. We present a case of Reiter's syndrome in a 55-year-old man who developed the typical skin lesions - kerathoderma blenorrhagicum. The disease started with a severe asymmetric oligoarthritis a month after the patient had urethritis. Two weeks after the onset of the arthritis red patches on the palms and plants appeared, which transformed quickly in harder and elevated plaques.

Key words: Syndroma Reiter, Keratoderma blenorrhagicum

\section{INTRODUCTION}

Reiter syndrome, also known as reactive arthritis, is a disease in which a non-suppurative polyarthritis lasting more then one month follows closely a lower urogenital or enteric infection. Classically it includes the triad arthritis, urethritis, and conjunctivitis. Approximately $80 \%$ of patients are positive for the histocompatability antigen called human leukocyte antigen (HLA)-B27; therefore, Reiter syndrome is strongly associated with HLA-B27. The disease is classified as type of seronegative spondyloarthropathy. Dermatologic manifestations are common, including keratoderma blenorrhagicum, circinate balanitis, nail changes and oral lesions.
\end{abstract}

\section{CASE REPORT}

\section{History}

We present a 55-year-old man admitted at a rheumatology department regarding a severe asymmetric oligoarthritis. The complaints appeared a month after outpatient was treated for urethritis. Two weeks after the onset of the acute arthritis the patient developed red patches on the palms and soles, which transformed quickly in harder and elevated plaques. The condition required a dermatological consultation. There were no data of internal diseases, enteral or respiratory infections. Family history was clear.

\section{Physical examination}

Physical examination in a dermatologic department a week after the appearance of the skin lesions revealed erythematous, confluent, hyperkeratotic papules and pseudopustules on the palms, soles, fingers and toes, and diffuse yellowish hyperkeratotic plaques on the soles (fig 1). Swelling and restricted movement of the left ankle and of the right wrist were observed (fig 2). There were no mucosal lesions, nail changes and other pathology from the physical examination including eye involvement.

\section{findings}

Lab and imaging studies, histopathological

The laboratory tests showed the following abnormalities: leukocytosis, elevated ESR and CRP, positive Chlamidia trachomatis antibodies $(\operatorname{IgA}(+)>1: 8$; $\operatorname{IgG}(+)$ 1:64). E. faecalis was isolated from the urine culture. RF was negative and the HLA-B27 positive. HIV was negative.

Hand and wrist X-ray did not show any abnormal findings. Abdominal sonography revealed hepatic steatosis and calculus in the right kidney.

Histopathology examination of the skin biopsy showed psoriasiform changes - hyper- and parakerathosis, acanthosis, elongation of the rete ridges in the epidermis and mixed inflammatory infiltrate in the upper dermis.

\section{Diagnosis}

The diagnosis Reiter syndrome was made according to the typical history, clinical symptoms and the findings of the above-mentioned laboratory tests. In this context the described skin lesions were interpreted as kerathoderma blenorrhagicum.

\section{Differential diagnosis}

Differential diagnosis of the articular involvement includes other seronegative arthritides - psoriatic arthritis, ankylosing spondylitis, gonococcal arthritis, rheumatoid arthritis. Skin involvement must be differentiated from pustular psoriasis, erythema multiforme, hyperkerathotic eczema of the palms and soles. 


\section{Therapy}

The patient underwent systemic therapy with corticosteroids, NSAID-s and antibiotics and local therapy with keratolytics and emollients.

\section{Clinical course}

The urogenital infection was successfully treated. A considerable improvement of the arthicular complaints was achieved two months after their occurrence.

The hyperkeratotic papules and plaques were gradually resolved and desquamated (fig 3). Complete clinical resolution of the skin lesions was observed one month after their occurrence (fig 4).

\section{DISCUSSION}

The name Reiter's syndrome was popularized in English texts by Bauer and Engelman in 1942, who thought that Reiter was the first to describe it $(1,5)$. At the same time of the disease's description by Reiter in 1916, two French doctors, Fiessinger and Leroy, attribute a case of reactive arthritis to a Shigella infection. In 1818 Brodie published a case of Reiter's syndrome after venereal infection, followed by Stoll, who described a case of the disease in $1869(1,5)$.

Classically Reiter's syndrome includes the triad urethritis, arthritis and conjunctivitis in young male patient after a urogenital or gastrointestinal infection. The disease is classified as a seronegativ spondyloarthropathy, because the patients are negative for rheumatoid factor.

The mechanism by which the organisms causing infection in the urogenital or gastrointestinal tract lead to Reiter's syndrome in some individuals is not clear. One hypothesis is cross-reactivity, in which antibodies developed against the inciting infection also have affinity for HLA-B27 (2). Another hypothesis involves cytokines. One study found decreased interleukin-2 production in HLA-B27 and B7-positive patients who developed reactive arthritis after Salmonella infection, suggesting that impaired cellular immunity against the inciting organism may correlate with development of the disease (4).

Diagnosing Reiter's disease can be difficult because of the great variation of the clinical features and because the classical triad is present in only one third of the patient (8). Usually, as in the case we present, the disease starts within 4 weeks after infection. Patients may experience any number of features, including generalized symptoms such as fever, fatigue, and weight loss, and urogenital, rheumatologic, ophthalmologic, dermatologic, and rarely visceral manifestations. Diagnosis is made by medical history and clinical findings, but symptoms taken individually cannot diagnose the disease - it become more evident with a constellation of symptoms. The American Rheumatism Association developed in 1981 the following diagnostic criteria for Reiter's disease: an episode of peripheral arthritis of more than 1-month duration occurring in association with urethritis, cervicitis, or both (6).

The incidence of mucocutaneous lesions in Reiter syndrome is variably reported between 8 and $31 \%$ of all cases. However a much lower incidence of about $1 \%$ has been attributed to post-dysenteric cases.

Cutaneous involvement includes psoriasiform lesions, named keratoderma blenorrhagicum (in about $10 \%$ ), painful and erosive lesions on the tips of the fingers and toes, nail dystrophy (20-30\%) and erythroderma as a rare complication. Erythematous macules and plaques, diffuse erythema, erosions, and bleeding can appear on the oral and pharyngeal mucosae. Lesions on glans penis, terms circinate balanitis (up to $50 \%$ ), are common and consist of vesicles that rupture to form painless superficial erosions.

Keratoderma blenorrhagicum is the characteristic cutaneous manifestation of the disease, although it occurs in only about $10 \%$ of patients. It usually appears $1-2$ months after the onset of arthritis as in our case, but may accompany or rarely precede the initial manifestations. The soles of the feet are almost always involved, but the extensor surfaces of the legs, the dorsal aspects of the toes, feet, hands, fingers, nails and scalp are common sites. In the case we present nail changes and lesions on the scalp were absent. Occasionally the eruption is very widespread and it may evolve into generalized exfoliative dermatitis (erythroderma). The initial lesion is a dull red macule which rapidly becomes papular and pseudovesicular. Its color changes from yellow to orange-red as the roof thickens to form a hyperkaratotic plaque. The mature lesion may stand out a centimeter or so from the skin surface. Keratoderma is often self-limiting, lasting weeks or months (3).

The clinical and histological features of Reiter's disease skin lesions are psoriasiform and fall within a spectrum of changes seen in idiopathic psoriasis. In view of the morphological similarities evolution into banal psoriasis, psoriatic arthritis and HLA studies, it seems possible that keratoderma represents the provocation of a psoriatic diathesis by the disease. Nevertheless the overall pattern, course and distribution of the eruption in Reiter's disease are usually distinctive $(3,7)$.

\section{CONCLUSION}

The case we presented shows the typical localization, morphologic features and clinical course of keratoderma blennorrhagicum as a characteristic cutaneous manifestation of Reiter syndrome. 

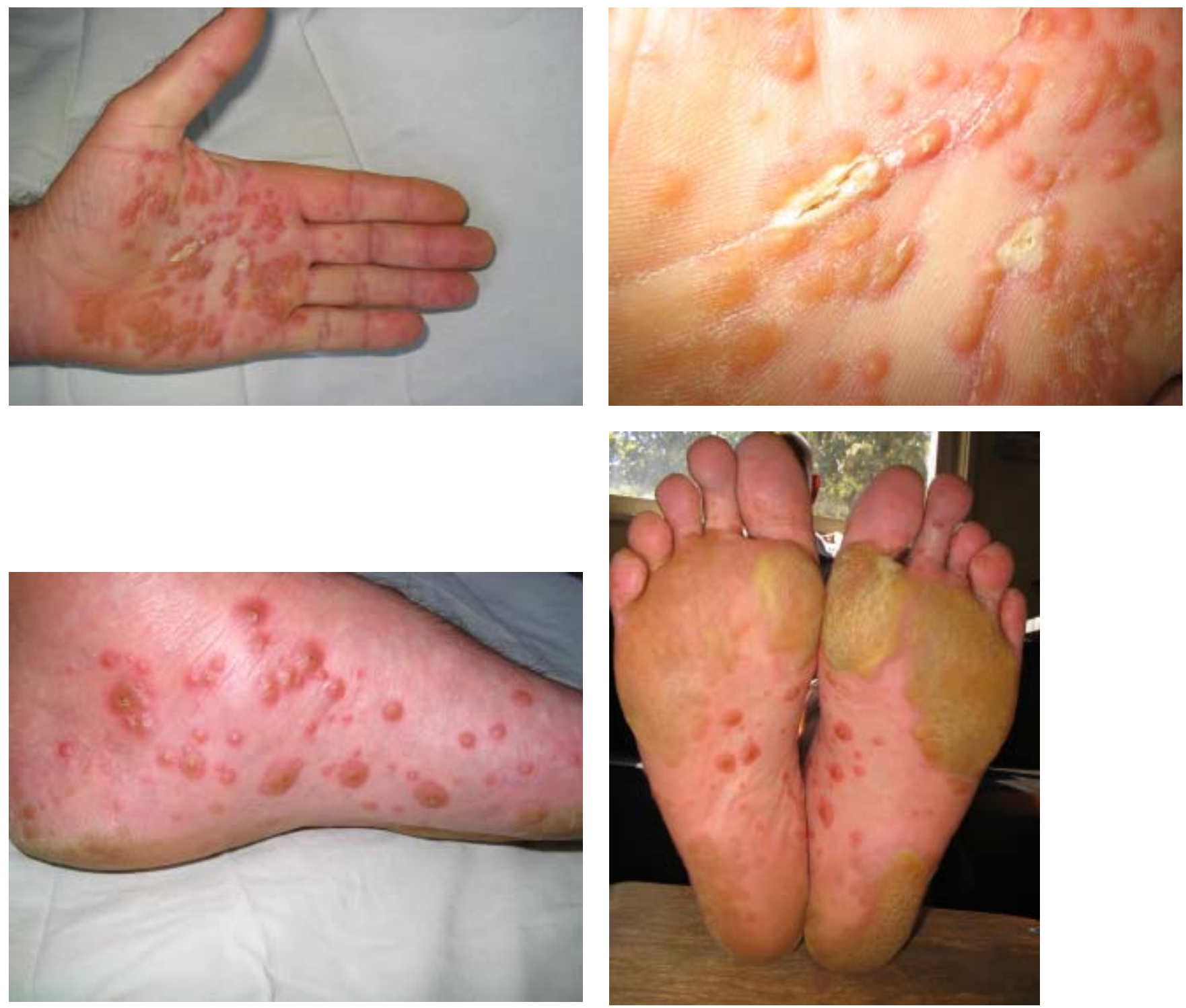

Fig. 1 (a, b, c, d): Keratoderma blenorrhagicum skin lesions a week after their appearance.

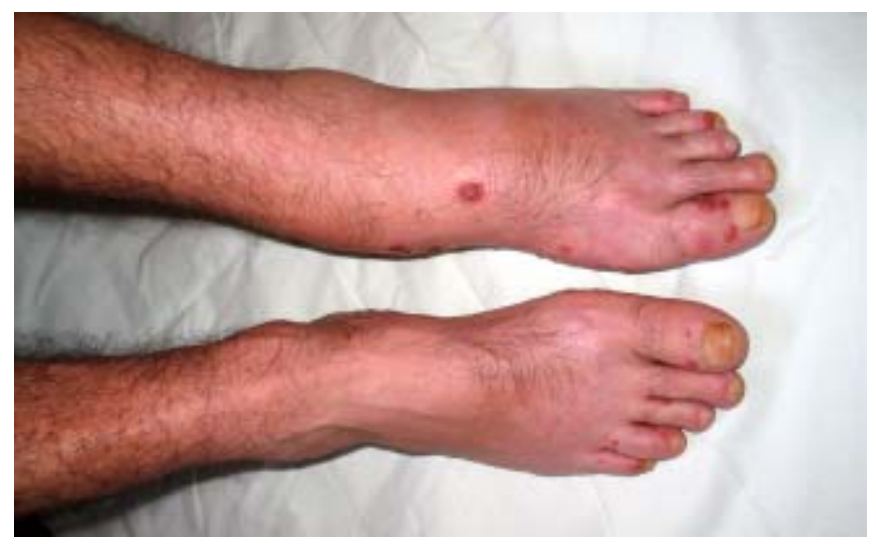

Fig. 2: Articular involvement - swelling of the left ankle. 

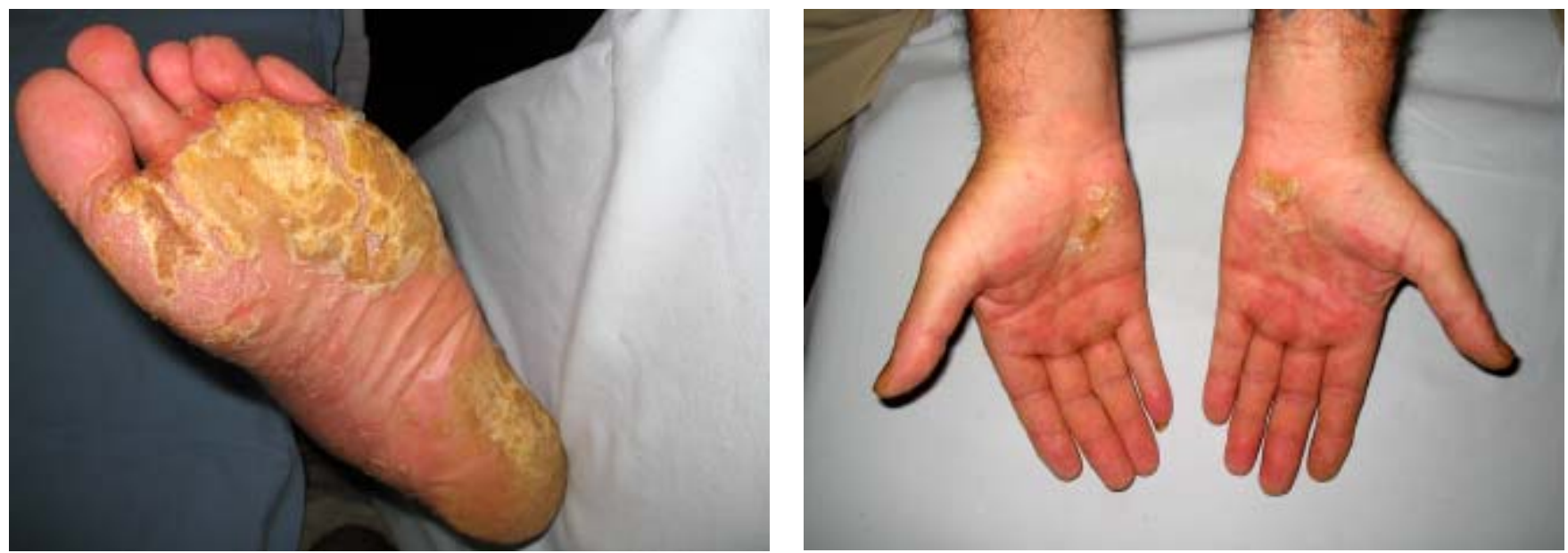

Fig. 3 (a, b): Initial clinical resolution of the skin lesions.
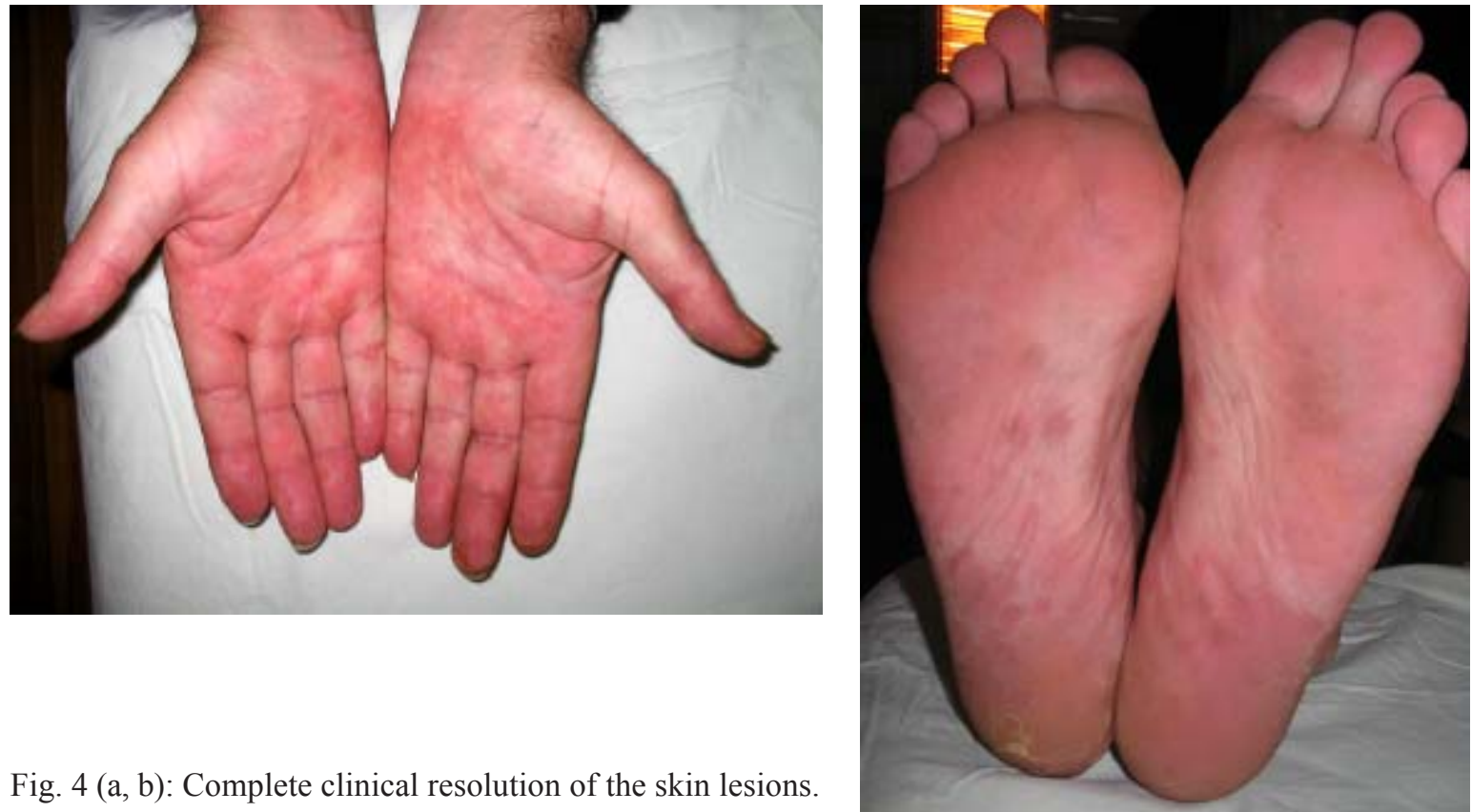

Fig. 4 (a, b): Complete clinical resolution of the skin lesions.

\section{BIBLIOGRAPHY:}

1. Anil Kumar Aribandi, Oludare Adetokundo Demuren, Reiter Syndrome, Musculoskeletal, Juli 2006 - article on eMedicine.

2. Eapen BR. A new insight into the pathogenesis of Reiter's syndrome using bioinformatics tools. Int J Dermatol 2003; 42:242-3.

3. Graham RM. Reiter's disease, Textbook of Dermatology, Rook/Wilkinson/
Ebling 1992, 5 edition.

4. Inman RD, Chiu B, Johnston ME, Vas S, Falk J. HLA class I-related impairment in IL-2 production and lymphocyte response to microbial antigens in reactive arthritis. J Immunol 1989; 142: 4256-60.

5. Schwartz RA, Romann J., Juli 2006, Reactive arthritis - article on eMedicine.

6. Willkens RF, Arnett FC, Bitter T,
Calin A, Fisher L, Ford DK, et al. Reiter's syndrome: evaluation of preliminary criteria for definite disease. Arthritis Rheum 1981; 24:844-9.

7. Wu IB., Schwartz RA. Reiter's syndrome: The classic triad and more. J Am Acad Dermatol 2008; 59: 113-21.

8. Rothe MJ, Kerdel FA. Reiter syndrome. Int J Dermatol 1991; 30: 17380 .

\section{Address for correspondence:}

Dr. Valentina Dimitrova

Department of Dermatology and Venereology, Medical University of Pleven 91, Gen. Vladimir Vazov str., 5800 Pleven, Bulgaria

Tel./Fax: +359 64886 622; E-mail: valentinadim@gmail.com 